\title{
Review
}

\section{Research Progress on Diagnosis Methods for Fungal Infection}

\author{
Hua Feng
}

Department of Emergency Medicine, Qingzhou TCM Hospital, Qingzhou China

\section{Keywords \\ Fungal infection diagnosis, G test, GM test, \\ PCR}

\section{Correspondence}

Hua Feng,

E-mail: huafqz@sina.com

DOI: $10.1515 / i-2017-0116$

\begin{abstract}
In recent years, owing to abuse of antibiotics, extensive use of antitumor drugs and immunosuppressive agents and other reasons, an increasing number of people suffered from fungal infection. In this situation, researchers proposed new diagnosis methods, such as $\mathrm{G}$ test, galactomannan (GM) test, and polymerase chain reaction (PCR). G test is simple, quick, and highly sensitive and can detect multiple fungi; however, it cannot distinguish fungal types and may result in false positive and false negative results. GM test is less time consuming and feature highly positive detection rates but can simply be used in inspection of invasive aspergillosis. However, optimal positive critical values of GM test remain controversial. PCR is currently one of the fastest methods but is not formally used in clinical practice because of its lack of standardized operation and evaluation criteria. This study reviews the above three methods with the aim of discovering and summarizing their advantages and disadvantages to facilitate research and development of new diagnosis methods.
\end{abstract}

\section{G test}

Prevalence rate and fatality rate of fungal infections increase globally because of the wide use of various antibiotics and development of technologies such as organ transplantation [1]. Depending on affected parts of the body, fungal infectious diseases can be divided into superficial fungal infections, skin fungal infections, subcutaneous fungal infections, and systemic fungal infections. Shallow fungal infections comprise superficial fungal infections and skin fungal infections, whereas invasive fungal infections include subcutaneous fungal infections and systemic fungal infections. Invasive fungal infection is significantly harmful and is an important type of hospital infection. Therefore, this study mainly describes research progress on diagnosis methods of invasive fungal infections. To date, common clinical diagnosis methods of fungal infections include mostly traditional methods, including direct smear methods, fungal culture methods, and pathological tissue examination ${ }^{[2]}$. However, given various factors, e.g., direct smear methods are less sensitive, pathological tissue examination traumatizes patients to some extent, and fungal culture method is time consuming ${ }^{[3]}$, these methods feature some limitations in their application in clinical practice, significantly affecting treatment of patients with fungal infection. Recently, methods, such as G test, galactomannan (GM) test, and polymerase chain reaction (PCR), were suggested for early diagnoses of domestic and foreign fungi. These methods improve speed and accuracy of early diagnoses of fungal infections, contribute to reasonably using antibacterial agents, and decrease mortality rate and resistance rate of bacteria.

$G$ test is mainly used to detect fungal cell wall composition, 1,3- $\beta$-D-mannan, which exists in cell wall of other deep fungi except for zygomycetes and cryptococcus. Deep fungi, including Candida and Aspergillus, are ingested and digested by phagocytes after they invade tissues, thus releasing 1,3- $\beta$-D-mannan. Some studies ${ }^{[4]}$ showed that 
$1,3-\beta$-D-mannan is barely released into the blood during fungal colonization and superficial fungal infections. However, this molecule occasionally exists in healthy human sera; thus, 1,3- $\beta$-D-mannan can be detected in organic blood, and sterile body fluids can be effective in diagnosis of deep fungal infections ${ }^{[s]}$. Dynamic monitoring data of $1,3-\beta$-D-mannan can serve as reliable indicator for evaluating efficacy of antifungal drugs and diseases ${ }^{[6]}$. The European Organization for the Research and Treatment of Cancer/ Mycology Study Group twice and consecutively considered $\mathrm{G}$ test as positive standard for clinical diagnosis of invasive fungal infectious diseases.

In relation to traditional fungal culture, $G$ test features simple and less time-consuming experimental conditions and operations. Specimen collection during result detection last for 3-4 h. G test can detect multiple fungi, such as Candida, Aspergillus, Pneumocystis, and Fusarium ${ }^{[7]}$, showing the highest sensitivity for Candida detection. Foreign studies indicated ${ }^{[8]}$ that sensitivity of $\mathrm{G}$ test to detection of invasive Candida reaches $47 \%-87 \%$. Metaanalysis of 14 studies for immunosuppressed patients with invasive fungal infectious diseases showed high sensitivity and specificity of G test ${ }^{[9]}$. $G$ test detects sera of patients with positive fungal culture and distinguishes colonization and infections, preventing unnecessary therapies to a large extent ${ }^{[10]}$. However, G test also presents some shortcomings.

First, G test cannot determine species, which poses less influence on clinical therapies because currently, clinical antifungal therapy does not vary with different species. Second, G test may yield false positive and false negative results. Fungi such as Aspergillus and Candida albicans are mostly normal floras in the human body and distributed on parts, such as the vagina and gut. Thus, fungi in those parts may release 1,3- $\beta$-D-mannan into the peripheral blood in reaction to local immune cells, resulting in increased percentages of false positive results ${ }^{[11]}$. False positive results also occur when glucans in food enter the blood, or some diseases cause an increase in glucans in the blood. Alternatively, some bacteria can produce 1,3- $\beta$-D-mannan, which causes false positives. A study ${ }^{[12]}$ showed that Gramnegative bacillary bacteremia is more apt to cause false positive results in $G$ test than Gram-positive bacillary bacteremia. As less 1,3- $\beta$-D-mannan is released, false negative results may be obtained.
To address failure of $\mathrm{G}$ test to determine species, joint detection can be carried out in $\mathrm{G}$ test and fungal culture; it contributes to determination of species and increases degree of sensitivity of diagnosis. For false positive phenomena in G test, detection can be conducted in conjunction with GM test, which increases sensitivity and specificity of $\mathrm{G}$ test to some extent and reduces probability of occurrence of false positives and false negatives ${ }^{[13]}$. However, fungal infections still cannot be disregarded when test results are all negative.

\section{GM test}

GM antigen detection is an effective method for early diagnosis of invasive aspergillosis. GM is a polysaccharide antigen on the cell wall of Aspergillus, and it is released into the blood during invasive infections. Days before clinical symptoms manifest in patients with Aspergillus infection, or when abnormality exists in imaging examinations, positive GM circulates the body ${ }^{[14]}$. GM can be detected with immunological test methods, including latex agglutination test, radioimmunoassay, enzyme-linked immunosorbentinhibition assay, and double-antibody sandwich enzymelinked immunosorbent assay. Double-antibody sandwich enzyme-linked immunosorbent assay is commonly used in clinical practice, and it is also an internationally recognized and highly sensitive detection method, which is widely used in Europe and America and approved by US FDA as auxiliary diagnosis indicator for clinical diagnosis of invasive Aspergillus infections.

Compared with imaging examination and direct microscopy, GM test is less time consuming and yielded higher positive detection rates. When anti-Aspergillus treatment is carried out in high-risk hematonosis patients with agranulocytosis according to a positive GM test, treatment efficacy rates can be increased. According to Luong et al. ${ }^{[15]}$, for bronchoalveolar lavage fluid (BALF) GM test used to diagnose invasive aspergillosis in patients with malignant hematopathy, sensitivity totals $100 \%$, and specificity reaches $87 \%$. BALF GM test is superior to direct microscopy, fungal culture, and blood GM test. Released amount of GM is proportional to the number of bacteria and thus can reflect infectious degree of patients. Dynamic monitoring of GM in clinically in high-risk patients enables 
early discovery of Aspergillus infections and timely treatment [16].

GM test features certain defects. First, GM test may yield false positive and false negative results to some extent. Some studies showed that use of $\beta$-lactams antibacterial drugs commonly causes false negative results in GM test. Therefore, prior to detection of Aspergillus galactomannan, care should be taken to avoid administration of medicine against this organism to patients. Low GM contents mainly cause false negative results in GM test. Second, positive cutoff values of GM test remain controversial. Most studies argued good sensitivity and specificity with single $\mathrm{I} \geq 1.0$ as a positive cutoff value. Other studies suggested that cutoff value of serum GM is $0.7 \mathrm{~h}$, and good comprehensive effects result from its sensitivity and specificity and predicted positive and negative values ${ }^{[17]}$.

\section{PCR}

PCR is a method in which a universal primer is designed in highly conserved regions of fungal rDNA. PCR amplifies target of rDNA and conducts sequencing on this product. This method is fast and flexible. Compared with conventional yeast culture methods, PCR can derive more accurate results in $24 \mathrm{~h}$. PCR can also be used in detection of mixed and unusual fungi ${ }^{[18]}$. However, as PCR presents false positive results and lacks standardized operation and determination criteria, US FDA does not approve use of PCR in clinical diagnosis of fungal infections ${ }^{[19]}$. Only one method is currently used in diagnosis of secondary pneumocystosis in patients with tuberculosis and HIV ${ }^{[20]}$.

In research of molecular biological methods for microbial identification and classification, selection of genes is crucial. PCR expansion can conduct sequencing for internal transcribed spacer (ITS)-1 region of fungal rDNA gene operons to identify species. Hypervariable sequence D1D2 regions of $8 \mathrm{~S}$ rDNA genes and $28 \mathrm{~S}$ rDNA genes are often used as target genes for PCR detection. D1-D2 and ITS regions of $28 \mathrm{~S}$ rDNA genes show significant variations in evolution. Between fungi with close genetic relationships, two gene regions also present significant variations. Thus, $28 \mathrm{~S}$ rDNA genes can serve as basis for identification between fungal species and variants or strains. Some studies [21] leveraged features of quick variation in mitochondrial
DNA, difference in evolution speeds of different regions, designed Candida albicans-specific primers based on fungal mitochondrial COII gene sequences, and analyzed sensitivity, specificity, and repeatability of fluorescent quantitative PCR. Results showed high sensitivity, strong specificity, and good repeatability and stability of using fluorescent PCR based on fungal mitochondrial COII genes in identification of Candida albicans.

With development of medical technologies, PCR constantly improved. To date, a range of related technologies were developed; these technologies include nested PCR, real-time quantitative PCR, and multi-PCR. Using realtime quantitative PCR allows fast and accurate diagnostic analysis of invasive aspergillosis and identification of species in patients' blood and BALF specimens, contributing to clinical and pertinent treatment with antifungal drugs ${ }^{[22,23]}$. Some studies compared two commercial PCR technologies, namely, Pneumocystis jirovecii PCR and Aspergillus PCR, with traditional diagnostic methods. Results showed better sensitivity and specificity of these commercial real-time PCR than those of traditional diagnostic methods ${ }^{[24]}$. For selection of blood and BALF specimens, some studies presented that fungal pathogens are much easily detected in mass sera and BALF drugs than in small amounts of sera and centrifuged BALF ${ }^{[25]}$. Ref. ${ }^{[26]}$ also showed that embedding specimens with paraffins significantly improves sensitivity of PCR in identifying yeasts and filamentous fungi. PCR using paraffins to embed specimens features high sensitivity. However, one remarkable weakness of this method is ubiquitous fungal contaminations in the environment ${ }^{[27]}$. Such fungal contaminations affect accuracy of detection and identification of fungi.

Real-time fluorescent quantitative PCR adds fluorophores in reaction system of PCR, uses fluorescence signals for real-time monitoring of the entire PCR, and finally conducts quantitative analysis of unknown templates through standard curves. This method is fast and show high sensitivity and good repeatability and can recognize species involved in infections ${ }^{[28]}$. However, this method is relatively expensive and may be limited in clinical application because of lack of appropriate real-time PCR devices and sequencing services. Multi-PCR uses multiple pairs of special primers to simultaneously amplify different target segments and detects multiple pathogens 
in one-time basis. To date, multi-PCR is used in detection [29] of Aspergillus, Mucor, and Rhizopus infections and joint examination ${ }^{[30]}$ of fungi and bacteria. Some researchers built quadruple PCR. With this technology, fungi are detected in approximately $5 \mathrm{~h}$, and species can be identified. However, quadruple PCR cannot determine colonization and infections and thus requires combination of clinical manifestation and traditional fungal diagnoses to carry out comprehensive analysis ${ }^{[31]}$.

To date, detection of Cryptococcus by real-time PCR is mostly based on amplification of single-copy genes, which may decrease sensitivity of real-time PCR ${ }^{[32]}$. Multi-real-time PCR (MRT-PCR) features high sensitivity and repeatability and can use a number of specimens, such as BALF, serums, and biopsy specimens, and allows for detection of mixed bacteria despite its failure to distinguish species, which pose less influence on clinical diagnoses and treatments ${ }^{[33]}$. MRTPCR is more expensive than traditional diagnostic methods, but it can detect infection of mixed bacteria and avoid delay in antifungal therapies ${ }^{[34]}$. MRT-PCR is superior to realtime PCR. However, additional specimen research are still necessary.

PCR technologies, real-time PCR or multi-PCR may produce false positive and false negative results during detection. Some studies showed that internal amplification control is an important factor in removal of false negatives. Alternatively, mutual authentication of results of detection systems of Aphanomyces spp. and Candida spp. can effectively reduce occurrence of false negatives and false positives ${ }^{[35]}$.

\section{Conclusions}

Fungal infections, especially Candida and Aspergillus deeporiented fungal infections, present high false negative rates and mortality rates and require costly clinical treatments; therefore, early diagnosis of fungi bears significance in addressing these conditions ${ }^{[36]}$. To date, traditional microscopy, fungal culture, and pathological tissue examination still prevail clinically. These methods are less sensitive and time consuming and detrimental to early detection of fungi and early treatment of fungal infections. To date, G test and GM test-oriented serological examinations and PCR-focused molecular examinations serve as research hotspots and achieved some progress but are still not widely used in clinical applications because of their respective limitations. Joint application of all methods must be considered. Theoretically, such combination not only can improve accuracy of major application methods but also can make up for certain deficiencies of auxiliary techniques. However, several specimens are still required for research on joint methods to ensure their correct clinical applications.

\section{Declarations}

\section{Acknowledgements}

No.

\section{Competing interests}

The author declares that he has no competing interest.

\section{Authors' contributions}

$\mathrm{H}$ Feng made the literature analysis and wrote, discussed and revised the manuscript of this review.

\section{References}

1 Olivier Brissaud, Julie Guichoux, Jerome Harambat, et al. Invasive fungal disease in PICU: epidemiology and risk factors. Annals of Intensive Care. 2012, 2(1):22.

2 Emmanuel Roilides. Invasive candidiasis in neonates and children. Early Human Development. 2011(87): S75-76.

3 Barton RC. Laboratory diagnosis of invasive aspergillosis: from diagnosis to prediction of outcome. Scientifica. 2013. http://dx.doi. org/10.1155/2013/459405.

4 Yasemin Oz, Nuri Kiraz. Diagnostic methods for fungal infections in pediatric patients: microbiological, serological and molecular methods. EXPERT REVIEW OF ANTI-INFECTIVE THERAPY. 2011, 9(3):289-98.

5 Sarika Jain, Shukla Das, Neelima Gupta, et al. Frequency of fungal isolation and antifungal susceptibility pattern of the fungal isolates from nasal polyps of chronic rhinosinusitis patients at a tertiary care centre in north India. Medical Mycology. 2013, 51(2):164-9.

6 Jaijakul S, Vazquez JA, Swanson RN, et al. $(1,3)-\mathrm{D}-$ Glucan as a Prognostic Marker of Treatment Response in Invasive Candidiasis. Clinical Infectious Diseases. 2012, 55(4):521-6.

7 Lu Y, Chen YQ, Guo YL. Diagnosis of Invasive Fungal Disease Using Serum (1,3)-beta-D-Glucan: A Bivariate Meta-Analysis. Journal of Internal Medicine. 2011, 50(22):2783-91. 
8 Lamoth F, Cruciani M, Mengoli C, et al. B-Glucan Antigenemia Assay for the Diagnosis of Invasive Fungal Infections in Patients With Hematological Malignancies: A Systematic Review and MetaAnalysis of Cohort Studies From the Third European Conference on Infections in Leukemia (ECIL-3). Clinical Infectious Diseases. 2012, (5):633-43.

9 Karageorgopoulos DE, Qu JM, Korbila IP, et al. Accuracy of $\beta$-D-glucan for the diagnosis of Pneumocystis jirovecii pneumonia: a meta-analysis. Clinical Microbiology and Infection. 2013, 19 (1):39-49.

10 Mortensen KL, Johansen HK, Fuursted K, et al. A prospective survey of Aspergillus spp. in respiratory tract samples: prevalence, clinical impact and antifungal susceptibility. European Journal. 2011, 30(11):1355-63.

11 Kobayashi K, Kimura S, Heux L, et al. Crystal transition between hydrate and anhydrous $(1 \rightarrow 3)-\beta$ - $d$-xylan from Penicillus dumetosus. Carbohydrate Polymers, 2013, 97 (1):105-110.

12 Albert O, Toubas D, Strady C, et al. Reactivity of $(1 \rightarrow 3)-\beta$-d-glucan assay in bacterial bloodstream infections. Eur J Clin Microbiol Infect Dis, $2011,30(11): 1453-60$.

13 Acosta J, Catalan M, Palacio-Pérez-Medel A, et al. Prospective study in critically ill non-neutropenic patients: diagnostic potential of $(1,3)-\beta$-D-glucan assay and circulating galactomannan for the diagnosis of invasive fungal disease. Eur J Clin Microbiol Infect Dis, 2012, 31(5): 721-31.

14 Arvanitis M, A nagnostou T, Fuchs BB, et al. Molecular and nonmolecular diagnostic methods for invasive fungal infections. Clinical Microbiology Reviews. 2014, 27(3):490-526.

15 Luong ML, Filion C, Labbé AC, et al. Clinical utility and prognostic value of bronchoalveolar lavage galactomannan in patients with hematologic malignancies. Diagnostic Microbiology \& Infectious Disease. 2010, 68(2):132-9.

Mikulsks M, Furfaro Em Del Bono V, er al. Galactomannan testing might be useful for early diagnosis of fusariosis. Diagnostic Microbiology \& Infectious Disease. 2012, 72(4):367-9.

17 Zhou X. Diagnosis value of serum galactomannan for detecting invasive pulmonary aspergillosis in patients with chronic. obstructive pulmonary disease. Clinical Education of General Practice, 2014,12(4):380-2.

18 Xafranski H, Melo AS, Machado AM, et al. A quick and low-cost PCRbased assay for Candida spp. identification in positive blood culture bottles. BMC Infectious Diseases. 2013, http://dx.doi.org/10.1186/14712334-13-467

19 Luis Ostrosky-Zeichner. Invasive Mycoses: Diagnostic Challenges. The American Journal of Medicine. 2012, 125(1Suppl): S14-24.

20 Boondireke S, Mungthin M, Tanariya P, et al. Evaluation of sensitivity of multiplex PCR for Detection of Mycobacterium tuberculosis and Pneumocystis jirovecii in clinical samples. Journal of Clinical
Microbiology. 2010, 48(9): 3165-8.

$21 \mathrm{Xu}$ F, He D, Sun X, et al. Establish a real-time PCR method to rapid identify Candida albicans. Chinese Journal of Laboratory Diagnosis, 2015, 19(4):528-31.

22 Zhao Y, Fu Y, Yin Z. The clinical value of assay of $(1,3)-\beta$-D-glucan in bronchoalveolar lavage fluid for invasive pulmonary fungal infection. Chinese journal of pathogen biology, 2015, 10(9):841-4.

23 Li S, Huang Y, Xu Y, et al. Detection of plasma (1-3) - $\beta$-D dextran in the diagnosis of deep fungal infection in patients with chemotherapy. Chinese journal of pathogen biology, 2011, 6(3):173-5,178.

24 Carlotta Francesca Orsi, Clotilde Bettua, Pietro Pini, et al. Detection of Pneumocystis jirovecii and Aspergillus spp.DNA in bronchoalveolar lavage fluids by commercial diagnostic tests. New Microbiologica. 2015, 38(1): $75-84$.

25

White P L, Bretagne S, Klingspor L, et al. Aspergillus PCR: one step closer to standardization. Journal of Clinical Microbiology. 2010, 48(4):1231-40. real time PCR with Sequencing and ribosomal RNA-FISH for the identification of fungi in formalin fixed, paraffin-embedded tissue specimens. BMC Infectious Diseases. 2011(11):201-13.

27 Babouee FB, Weisser M, Prince SS, et al. Performances of two different panfungal PCRs to detect mould DNA in formalin-fixed paraffinembedded tissue:what are the limiting factors?. BMC Infectious Diseases. 2014(14):692. Buitrago MJ, Bernal-Martinez L, Castelli MV, et al. Performance of panfungal and specific-PCR-based procedures for etiological diagnosis of invasive fungal diseases on tissue biopsy specimens with proven infection: a 7-year retrospective analysis from a reference laboratory. Journal of Clinical Microbiology. 2014, 52(5): 1737-40.

29 Development of a single tube multiplex real-time PCR to detect the most clinically relevant Mucormycetes species. L. Bernal-Martínez, M. J. Buitrago, M. V. Castelli, et al. Clinical Microbiology and Infection. 2013, 19(1): E1-7.

30 Gago S, Esteban C, Valero C, et al. A multiplex real-time PCR for the identification of Pneumocystis jirovecii,Histoplasma capsulatum and Cryptococcus neoformans/gattii causing opportunistic pneumonia in AIDS patients. J Clin Microbiol. 2014, 52(4): 1168-76.

31 Liu Z, Shao Y, Yang Y, et al. Establishment and clinical application of fungal quadruple PCR system. Chinese Journal of Dermatology and Venereology, 2015 ,29(5):539-43.

32 Qishui O, Ling J, Ni L, et al. Comparison of real-time florescence quantitative PCR measurements of VAD1 mRNA with three conventional methods in diagnosis and follow-up treatment of Cryptococcus neoformans infection. Mycoses. 2012, 55(4):326-32.

33 Antinori S. New insights into HIV/AIDS-associated cryptococcosis. 
ISRN AIDS, 2013(12):471363.

34 Gago S, Esteban C, Valero C, et al. A multiplex real-time PCR for the identification of Pneumocystis jirovecii,Histoplasma capsulatum and Cryptococcus neoformans/gattii causing opportunistic pneumonia in AIDS patients. J Clin Microbiol. 2014, 52(4):1168-76.

35 Li Y, Hu Z. Application of molecular biology techniques in diagnosis and treatment of invasive fungal infection. Chinese Journal of Mycology, $2014,9(6): 377-80$

36 Wang B, Wei M, Fang H, et al. New Advances in Drug Resistance Mechanism of Candida. Chinese journal of pathogen biology, 2014, $9(5): 473-7$. 